\title{
Editorial
}

\section{HIP Fracture Evaluation and Management}

\author{
Sumon Nandi $\left(\mathbb{D},{ }^{1}\right.$ Paul Dougherty, ${ }^{2}$ Gary Gruen, ${ }^{3}$ and Nabil Ebraheim $\mathbb{D}^{1}$ \\ ${ }^{1}$ The University of Toledo College of Medicine and Life Sciences, Toledo, OH, USA \\ ${ }^{2}$ The University of Florida College of Medicine-Jacksonville, Jacksonville, FL, USA \\ ${ }^{3}$ University of Pittsburgh, Pittsburgh, PA, USA \\ Correspondence should be addressed to Nabil Ebraheim; nabil.ebraheim@utoledo.edu \\ Received 8 November 2019; Accepted 9 November 2019; Published 17 December 2019 \\ Copyright (C) 2019 Sumon Nandi et al. This is an open access article distributed under the Creative Commons Attribution License, \\ which permits unrestricted use, distribution, and reproduction in any medium, provided the original work is properly cited.
}

Hip fracture incidence is over 250,000 per year in the United States and is expected to grow to 850,000 by the year 2040 [1]. The severity of a hip fracture is underscored by the mortality rate of 20 to $30 \%$ in the year following injury $[1,2]$. Most hip fractures occur in those older than 50, a demographic in which there is a higher prevalence of comorbidities [3]. As a result, minimizing perioperative adverse events while maximizing postoperative function and reoperation-free survival is essential in hip fracture patients. These aims not only seek to optimize quality of care in a large patient population, but also control cost as hip fractures comprise the majority of orthopaedic trauma-associated health care expenditures [4].

In this issue, investigators discuss preoperative optimization, intraoperative surgical technique, discharge planning, and management of postoperative complications in hip fracture patients. By offering guidance to surgeons throughout the entire episode of care, from hospital admission to follow-up, we hope to provide a broad-based overview of hip fracture evaluation and management.

T. S. Moores and colleagues describe an effective protocol for reversing the effect of warfarin in hip fracture patients that decreases time to the operating room (OR) following presentation. Morbidity and mortality following hip fracture are mitigated by decreasing time to OR following injury, so Moores' findings are impactful.

Subtrochanteric femur fractures pose a clinical challenge, not only due to the technical difficulty of reduction and fixation, but also the high rate of nonunion. C. Jackson et al.'s text offers technical pearls within the framework of an anatomic and biomechanical discussion that is helpful to surgeons as they strive to minimize these issues.
S. R. Konda and his coauthors present use of a validated risk assessment tool to predict discharge disposition and readmission among hip fracture patients. Strategies for optimization of discharge planning are offered to minimize costs associated with the hip fracture episode of care.

Nonunion following hip fracture fixation in the elderly is highly consequential as revision surgery may be required in this medically frail and functionally compromised patient population. J. F. Kellam et al. provide a discussion of optimal methods for hip fracture fixation to prevent nonunion, a rubric for determining the cause of nonunion, and revision surgery techniques to treat nonunion.

Nearly all orthopaedic surgeons care for hip fracture patients. In the issue to follow, we aim to present papers that are immediately applicable to the clinical practices of our broad audience.

\section{Conflicts of Interest}

The editors declare that there are no relevant conflicts of interest.

Sumon Nandi

Paul Dougherty

Gary Gruen

Nabil Ebraheim

\section{References}

[1] S. M. Friedman and D. A. Mendelson, "Epidemiology of fragility fractures," Clinics in Geriatric Medicine, vol. 30, no. 2, pp. 175-181, 2014. 
[2] O. Johnell and J. Kanis, "Epidemiology of osteoporotic fractures," Osteoporosis International, vol. 16, no. S02, pp. S3-S7, 2005.

[3] J. D. Zuckerman, "Hip fracture," New England Journal of Medicine, vol. 334, no. 23, pp. 1519-1525, 1996.

[4] N. F. Ray, J. K. Chan, M. Thamer, and L. J. Melton III, "Medical expenditures for the treatment of osteoporotic fractures in the United States in 1995: report from the National Osteoporosis Foundation," Journal of Bone and Mineral Research, vol. 12, no. 1, pp. 24-35, 1997. 


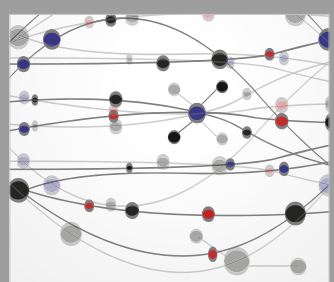

The Scientific World Journal
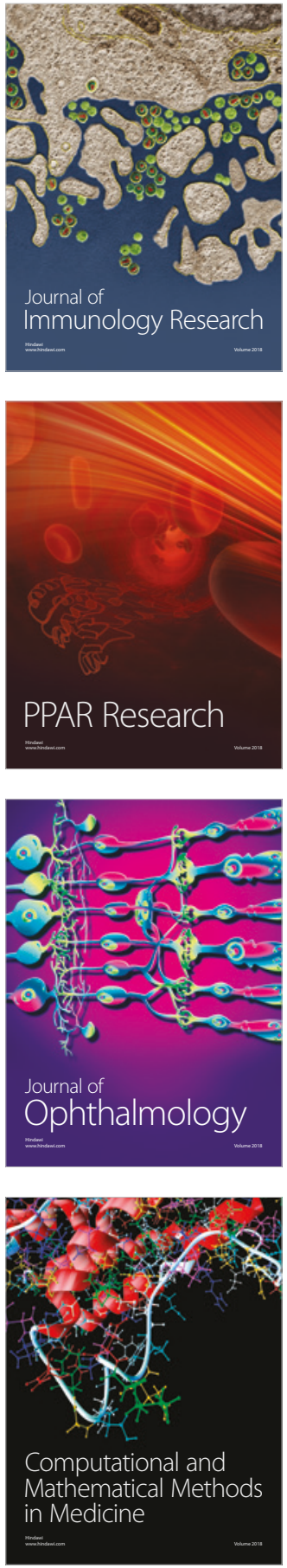

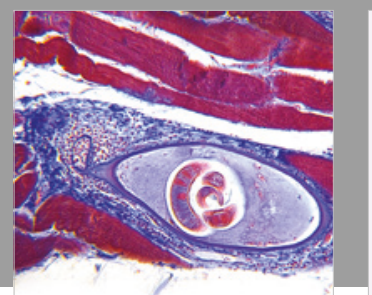

Gastroenterology Research and Practice

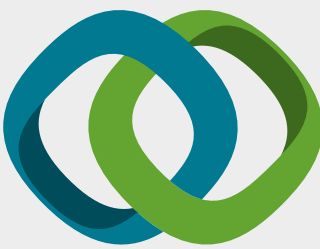

\section{Hindawi}

Submit your manuscripts at

www.hindawi.com
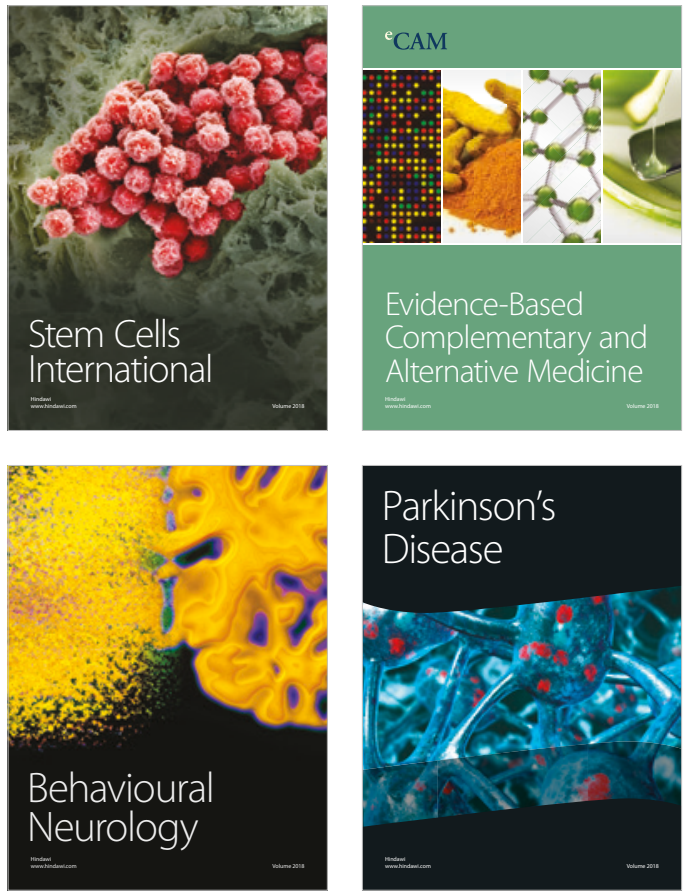

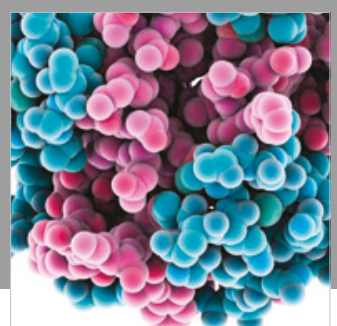

ournal of

Diabetes Research

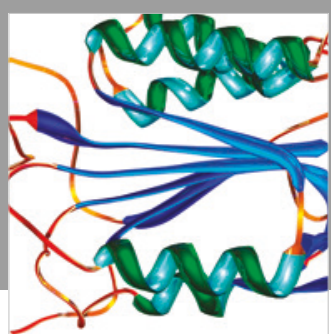

Disease Markers
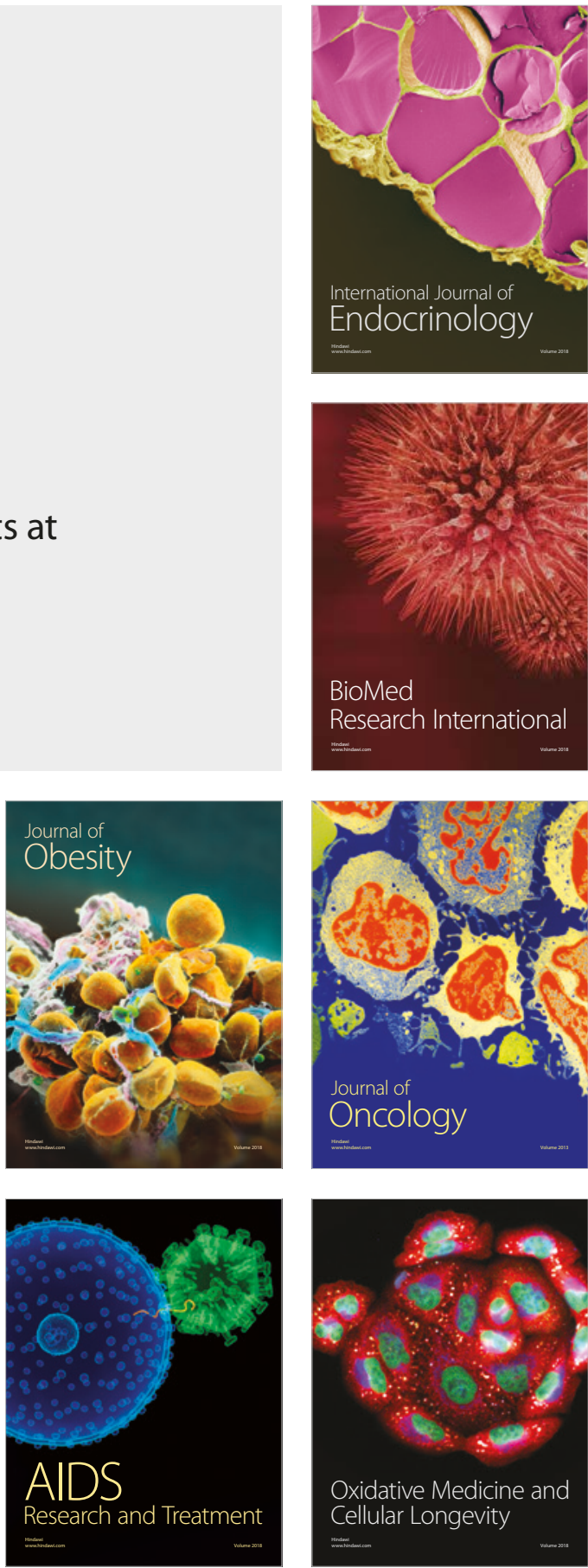